\title{
Genome-wide identification and characterization of LRR-RLKs reveal functional conservation of the SIF subfamily in cotton (Gossypium hirsutum)
}

Ning Yuan ${ }^{1}$, Krishan Mohan Rai ${ }^{1}$, Vimal Kumar Balasubramanian ${ }^{1}$, Santosh Kumar Upadhyay ${ }^{2}$, Hong Luo ${ }^{3}$ and Venugopal Mendu ${ }^{*^{*}}$ D

\begin{abstract}
Background: As one of the largest subfamilies of the receptor-like protein kinases (RLKS) in plants, Leucine Rich Repeats-RLKs (LRR-RLKs) are involved in many critical biological processes including growth, development and stress responses in addition to various physiological roles. Arabidopsis contains 234 LRR-RLKs, and four members of Stress Induced Factor (SIF) subfamily (AtSIF1-AtSIF4) which are involved in abiotic and biotic stress responses. Herein, we aimed at identification and functional characterization of SIF subfamily in cultivated tetraploid cotton Gossypium hirsutum.

Results: Genome-wide analysis of cotton LRR-RLK gene family identified 543 members and phylogenetic analysis led to the identification of 6 cotton LRR-RLKs with high homology to Arabidopsis SIFs. Of the six SIF homologs, GhSIF1 is highly conserved exhibiting $46-47 \%$ of homology with AtSIF subfamily in amino acid sequence. The GhSIF1 was transiently silenced using Virus-Induced Gene Silencing system specifically targeting the 3' Untranslated Region. The transiently silenced cotton seedlings showed enhanced salt tolerance compared to the control plants. Further, the transiently silenced plants showed better growth, lower electrolyte leakage, and higher chlorophyll and biomass contents.

Conclusions: Overall, 543 LRR-RLK genes were identified using genome-wide analysis in cultivated tetraploid cotton G. hirsutum. The present investigation also demonstrated the conserved salt tolerance function of SIF family member in cotton. The GhSIF1 gene can be knocked out using genome editing technologies to improve salt tolerance in cotton.
\end{abstract}

Keywords: Gossypium hirsutum, LRR-RLKs, Genome-wide analysis, Salt tolerance

\section{Background}

In order to sense outside environment and efficiently communicate between cells, both animals and plants use plasma membrane and/or cell wall localized receptors, which perceive and transduce signals to modulate gene expression. Toll-like receptors represent the most important kinase receptors involved in signal transduction process [1]. Plant receptor-like protein kinases (RLKs), on the other hand, is the most important membrane

\footnotetext{
* Correspondence: venugopal.mendu@ttu.edu

${ }^{1}$ Fiber and Biopolymer Research Institute (FBRI), Department of Plant and Soil Science, Texas Tech University, Lubbock, TX 79409, USA

Full list of author information is available at the end of the article
}

protein family involved in growth and development, stress response and various other biological processes [2]. Based on the structure of an extracellular domain, plant receptor-like protein kinases have been classified into various subfamilies such as S-RLK (S-domain RLK), LRR-RLK (Leucine-Rich Repeat RLK), CR4-class (CRINKLY4 RLK), WAK (Wall Associated Kinase), PR5-RLK (PR5-Like RLK), and Lectin class [3-9]. Among them, LRR-RLK is one of the largest subfamilies of the receptor-like protein kinases in plants with 234 members in Arabidopsis [2, 10-12] (Table 1). LRR domain specifically identifies and interacts with a wide variety of extracellular signaling ligands, conferring LRR-RLK's

(C) The Author(s). 2018 Open Access This article is distributed under the terms of the Creative Commons Attribution 4.0 International License (http://creativecommons.org/licenses/by/4.0/), which permits unrestricted use, distribution, and 
Table 1 Gene distribution comparison of Arabidopsis and cotton LRR-RLK subclades

\begin{tabular}{|c|c|c|c|c|c|}
\hline Subclade & G. hirsutum & Ratio in the total LRR-RLKs & Subclade & A. thaliana & Ratio in the total LRR-RLKs \\
\hline I & 13 & $2.4 \%$ & I & 44 & $18.8 \%$ \\
\hline$\|$ & 28 & $5.2 \%$ & $\|$ & 15 & $6.4 \%$ \\
\hline III & 89 & $16.4 \%$ & III & 45 & $19.2 \%$ \\
\hline IV (1 \&2) & 12 & $2.2 \%$ & IV & 4 & $1.7 \%$ \\
\hline V & 18 & $3.3 \%$ & V & 10 & $4.3 \%$ \\
\hline VI (1 \&2) & 30 & $5.5 \%$ & $\mathrm{VI}(1 \& 2)$ & 14 & $6.0 \%$ \\
\hline VII & 17 & $3.1 \%$ & VII & 7 & $3.0 \%$ \\
\hline VIIII (1 \&2) & 40 & $7.4 \%$ & VIII & 21 & $9.0 \%$ \\
\hline IX & 18 & $3.3 \%$ & IX & 5 & $2.1 \%$ \\
\hline$X(1,2,3 \& 4)$ & 43 & $7.9 \%$ & $x(a \& b)$ & 18 & $7.7 \%$ \\
\hline$X I(1,2,3 \& 4)$ & 93 & $17.1 \%$ & $X I$ & 30 & $12.8 \%$ \\
\hline XII (1 \& 2) & 128 & $23.6 \%$ & $X \|$ & 10 & $4.3 \%$ \\
\hline XIII & 13 & $2.4 \%$ & XIII (a \& b) & 10 & $4.3 \%$ \\
\hline Others & 1 & $0.2 \%$ & $\mathrm{XVl}$ & 1 & $0.4 \%$ \\
\hline Total & 543 & & & 234 & \\
\hline
\end{tabular}

ability to perceive apoplastic signals [13]. Studies on the FLS2 (Flagellin Sensitive 2)-BAK1 (Brassinosteroid Insensitive 1-associated receptor kinase 1) complex showed that the interaction between ligand and LRR domain induces a conformational change of kinase domain in the cytoplasm, which allows the kinase domain to transfer phosphates to downstream proteins, promoting the signal transduction from apoplast to symplast [13]. LRR-RLKs regulate various biological processes in plants, including steroid perception, cell proliferation, photomorphogenesis, biotic and abiotic stress responses [14-19]. For instance, SERKs (Somatic Embryogenesis Receptor Kinase) are essential receptors mediating brassinosteroid signal perception in Arabidopsis [20, 21]. Furthermore, SERK3/BAK1 and SERK4/BKK1 (BAK1-Like 1) are involved in defense signal transduction triggered by FLS2 or EFR [22]. In Medicago spp., the LRR-RLK gene, SRLK has been shown to regulate the root response to salt stress [18]. Similarly, rice Xa21D gene encodes a membrane-anchored protein responsible for the pathogen recognition in disease resistance signaling pathway [23].

Due to the significant importance of the LRR-RLK family members, genome-wide analysis has been performed in Arabidopsis, soybean, wheat, citrus, vernicia, maize, rice and poplar, facilitating identification and functional characterization of LRR-RLK genes in these species [12, 24-30]. LRR-RLKs in Arabidopsis are grouped into 14 subclades (LRR-I to LRR-XIV, which are distributed among all five chromosomes [12]. A total of 309, 467 and 531 LRR-RLKs have been identified in rice, soybean and allohexaploid wheat, respectively [24, 28, 30]. Despite the large numbers, the LRR-RLKs are highly conserved within the clades. However, differences in extracellular domains and the associated structure resulted in the functional specialization of individual members within the clades. For instance, Arabidopsis LRR-RLKs from subclade I harbor a malectin-like domain responsible for $N$-glycosylation and ER localization, which is not detected in other subclades [31]. Hence, phylogenetic analysis and functional characterization of each gene are important to understand their specific role in various organisms. We have recently identified and characterized a sub-family of LRR-RLK genes involved in biotic and abiotic stress signaling pathway in Arabidopsis [32]. The Stress Induced Factor (SIF) sub-family contains four members (SIF1-4), which respond to abiotic and biotic stresses. Further characterization of SIF2 protein demonstrated its role in stress signal transduction pathway in Arabidopsis.

Gossypium hirsutum is one of the widely cultivated crops in the world, which accounts for more than 95\% annual global cotton production [33]. Globally, cotton is cultivated under diverse environmental conditions and exposed to various biotic and abiotic stresses. Individual cotton LRR-RLKs genes, such as GhLRR-RL, GhBRI1, GhRLK1, and GbRLK, have been characterized and demonstrated to play important roles in cotton development and stress resistance [34-37]. However, there is no comprehensive analysis of the LRR-RLK gene family in cotton. In the present study, we performed genome-wide analysis of LRR-RLK gene family in G. hirsutum using the recently released cotton full genome sequence (https://www.cottongen.org/data/download/genome). A total of 543 GhLRRRLK proteins were identified, and 542 of them were grouped into 13 clades in a phylogenic tree. Chromosomal distribution, gene duplication, gene and protein structure 
analysis, functional annotation, and expression profiling of these genes further led to the identification of Arabidopsis SIF subfamily of homologs in cotton. Transient silencing of GhSIF1 using virus-induced gene silencing (VIGS) system conferred salt tolerance in cultivated tetraploid cotton. Overall, the present study demonstrates the functional conservation of SIF sub-family in cotton, suggesting its potential use for crop improvement through molecular breeding, biotechnology or genome editing approaches.

\section{Results \\ Identification of $L R R-R L K$ gene family in Gossypium hirsutum TM-1}

We have downloaded publicly available G. hirsutum TM-1 accession reference genome data and performed genome-wide similarity search to identify the LRR-RLK gene family using the sequences of Arabidopsis LRR-RLK proteins as query [12]. A stringent filtration of the Blast identified sequences for the presence of a minimum of one LRR repeat, a kinase domain and a transmembrane region resulted in identification of a total of $543 \mathrm{G}$. hirsutum LRR-RLK family members (Additional file 1: Table S1). Full-length genomic, coding and amino acid sequences for all the validated G. hirsutum LRR-RLK family members were fetched from the reference genome sequence with their original gene ID and used for further characterization.

\section{Phylogenetic analysis of cotton LRR-RLKs}

Protein sequence alignment and phylogenetic analysis were performed using 543 GhLRR-RLK and 234 Arabidopsis LRR-RLK protein sequences to study the evolutionary relationships $[11,12]$. G. hirsutum protein sequences that were grouped with AtLRR-RLK were defined as members of the corresponding Arabidopsis subclade. Using the Arabidopsis LRR-RLKs as references, 542 GhLRR-RLKs were grouped into 13 subclades in the Neighbor-Joining phylogenetic tree, while remaining one protein, CotAD_01838, was clustered together with an Arabidopsis LRR receptor-like protein At1G65380 (CLV2), which was not assigned to any Arabidopsis subclade (Fig. 1 \& Additional file 1: Table S1). The size of each GhLRR-RLK subclade varied significantly. For instance, the largest subclade XII contains 128 members, while the smallest subclade IV contains only 12 members. Broadly, the relative size of each GhLRR-RLK subclade was almost similar to Arabidopsis, except subclade I and subclade XII (Table 1) [38]. In Arabidopsis, subclade I has 44 members representing $18.8 \%$ of the total AtLRR-RLKs, but G. hirsutum subclade I, which contains 13 members comprises only $2.4 \%$ of the total GhLRR-RLKs. The subclade XII, 10 LRR-RLK sequences represent only $4.3 \%$ of the total AtLRR-RLKs, while GhLRR-RLK-XII subclade is composed of 128 members representing $23.6 \%$ of the total GhLRR-RLKs.

To investigate whether G. hirsutum contains homologs of Arabidopsis SIF subfamily genes (AtSIF1-AtSIF4) [32], we generated a Maximum Likelihood phylogenetic tree using AtSIF1-AtSIF4 proteins with G. hirsutum subclade I LRR-RLKs proteins which showed high homology with AtSIF2 (At1G51850) (Fig. 2). The phylogenetic tree showed that 9 GhLRR-RLKs have very close evolutionary relationship with the four Arabidopsis LRR-RLKs (Fig. 2a). Among these 9 GhLRR-RLKs, one cotton LRR-RLK (CotAD_41732) showed very high homology with AtSIF subfamily (Fig. 2a). To further understand the protein conservation between AtSIFs and the nine cotton LRRRLKs, multiple sequence analysis was performed (Fig. 2 \& Additional file 2: Data S1). The result showed that only six proteins out of the 9 GhLRR-RLKs contain the Malectin-like domain, which is also present in AtSIFs (Fig. 2b). LRR domain is one of the most critical domains in LRR-RLKs as it offers LRR-RLKs the ability of ligand recognition and interaction [39]. Highly conserved LRR domains in LRR-RLKs usually indicate functional conservation [39]. The amino acid sequence comparison of the LRR domains in these six LRR-RLKs which contain Malectin-like domain showed that CotAD_41732 exhibited the highest similarity with the AtSIFs, as it contains two highly conserved LRR motifs in the same region of the extracellular domains (Fig. 2c). Other cotton LRR-RLKs contain either different number of LRR motifs (such as CotAD_57195, CotAD_44233, CotAD_52119, CotAD_31444) or gaps in the critical LRR domains (such as CotAD_74481, CotAD_06671), or the size is significantly shorter than that of the AtSIFs (such as CotAD_21855 and CotAD_74959) (Fig. 2c). We, therefore, refer CotAD_41732 which showed highest similarity as GhSIF1 hereafter.

\section{Chromosomal distribution of GhLRR-RLKs}

To further investigate the evolutionary history of GhSIF1 as well as other GhLRR-RLKs, we analyzed their chromosomal distribution on both A and D subgenomes of G. hirsutum (Fig. 3 \& Additional file 1: Table S2). The GhLRR-RLK genes were distributed on all chromosomes of both subgenomes but at a different frequency (Fig. 3). Out of 543 genes, 179 and 219 genes could be confirmed at A and D subgenomes, respectively; whereas 145 genes were located on scaffolds (Additional file 3: Figure S1). A maximum of 32 and 46 genes and a minimum of one and three genes were located on chromosome 9 and chromosome 4 of A and D-subgenomes, respectively (Fig. 3 \& Additional file 3: Figure S1). GhSIF1 was located on the scaffold 1841.1 (Additional file 3: Figure S1 and Additional file 1: Table S2).

A total of 42 tandem duplication events (TDEs) were identified involving 110 genes distributed in subclades II, 


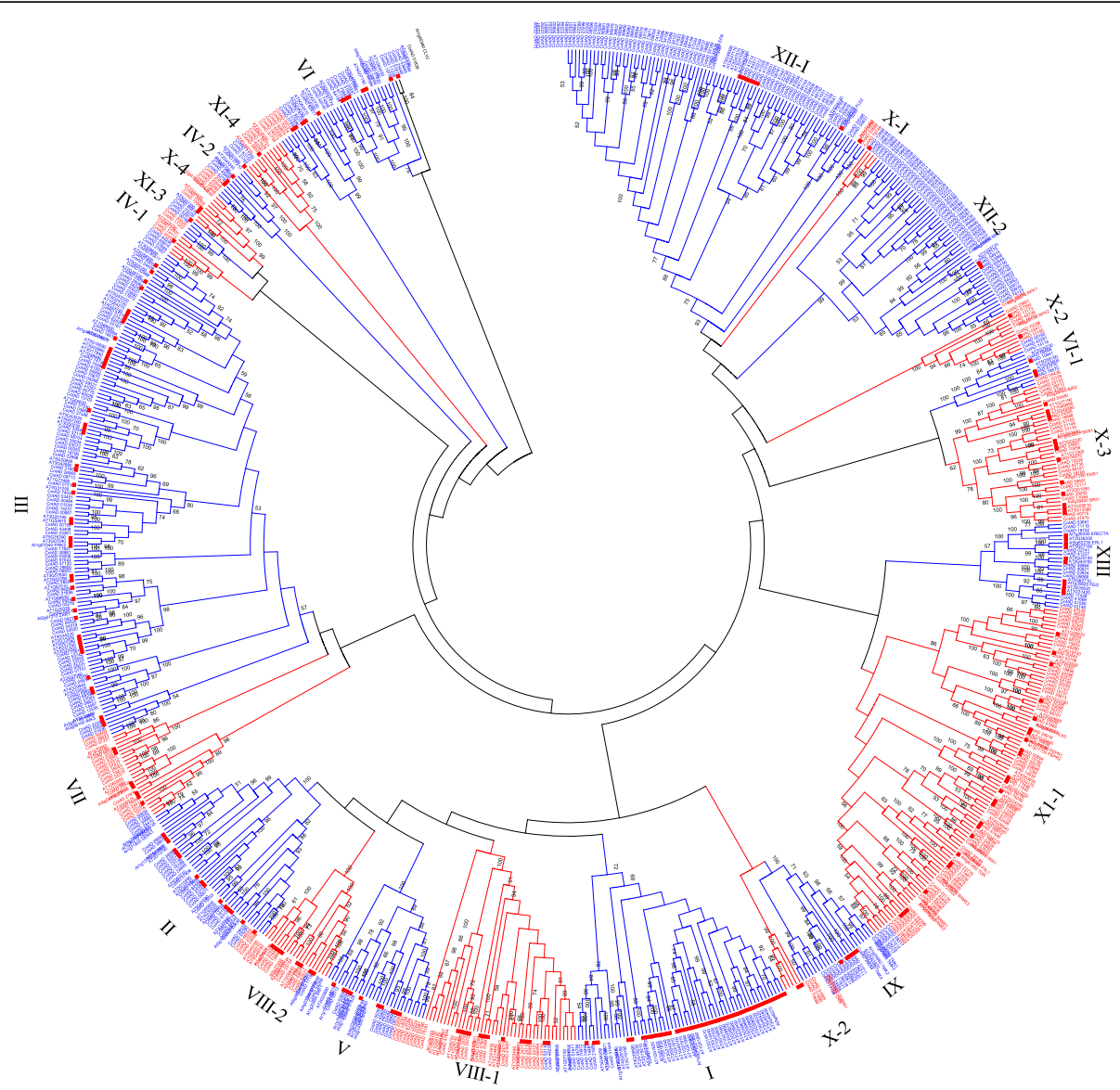

Fig. 1 Phylogenetic analysis of Gossypium hirsutum LRR-RLK protein sequences. The evolutionary history was inferred using the Neighbor-Joining method with 1000 bootstrap replication. The evolutionary distances were computed using the $p$-distance method and are in the units of the number of amino acid substitutions per site. The analysis involved 543 G. hirsutum LRR-RLK protein sequences and 234 Arabidopsis thaliana LRR-RLK protein sequences. All positions containing gaps and missing data were eliminated. Evolutionary analyses were conducted in MEGA6

III, VIII_1, X_4, XI_1, XII_1 and XII_2 (Fig. 3). Subclade XII_1 showed a maximum of 14 events involving 40 genes followed by subclade XII_2 with 12 events involving 32 genes. Out of 42 TDEs, 13 were observed on 8 chromosomes (Chr. 3, 5, 6, 8, 9, 10, 12 and 13) of A-subgenome (Fig. 3), while 15 were found on 8 chromosomes (Chr. 1, 3, $5,6,9,10,11$ and 13) of D-subgenome. The remaining 14 TDEs were observed on 10 unassigned scaffolds (Scaffold 2911.1 with three duplication events and scaffold 235.1 and 3068.1 with two events each). Overall, the analysis showed a high proportion of tandem duplications involving $\sim 1 / 5$ th of the LRR-RLKs.

\section{Analysis of gene structure (exon-intron organization) of GhLRR-RLKs}

Exon-intron structures of 543 GhLRR-RLK genes, including the GhSIF1, were analyzed and organized in different groups according to their subclades. As shown in Additional file 3: Figure S2 (A-I), the exon-intron organization of $L R R-R L K$ genes showed high variation among subclades, whereas, within subclade the genes displayed comparable structure in terms of number, size and position of exons. The conservation of gene structure within clades indicates that the $L R R-R L K$ genes within clades indeed have very close evolutionary relationships in the phylogenetic tree. Based on exon-intron structures, the GhLRR-RLKs could be classified into three groups (Additional file 3: Figure S2 A-I). All the members of subclade I, II, V, VI-2, VIII (1 \& 2 ), and most members of subclade XIII comprised multiple but relatively short exons, while the members of subclade III, IV (1 \& 2), VI-1, VII, IX, X (1-4), XI (1-4), two members of subclade XIII and CotAD 01838 consisted of several long exons. Subclade XII $(1 \& 2)$ genes showed a unique pattern with the combination of long exons and short exons.

\section{Protein structure analysis}

GhLRR-RLKs showed a wide variation in their length ranging from 234 to 1878 amino acid residues (aa) (Additional file 3: Figure S3 \& Additional file 1: Table S1) 


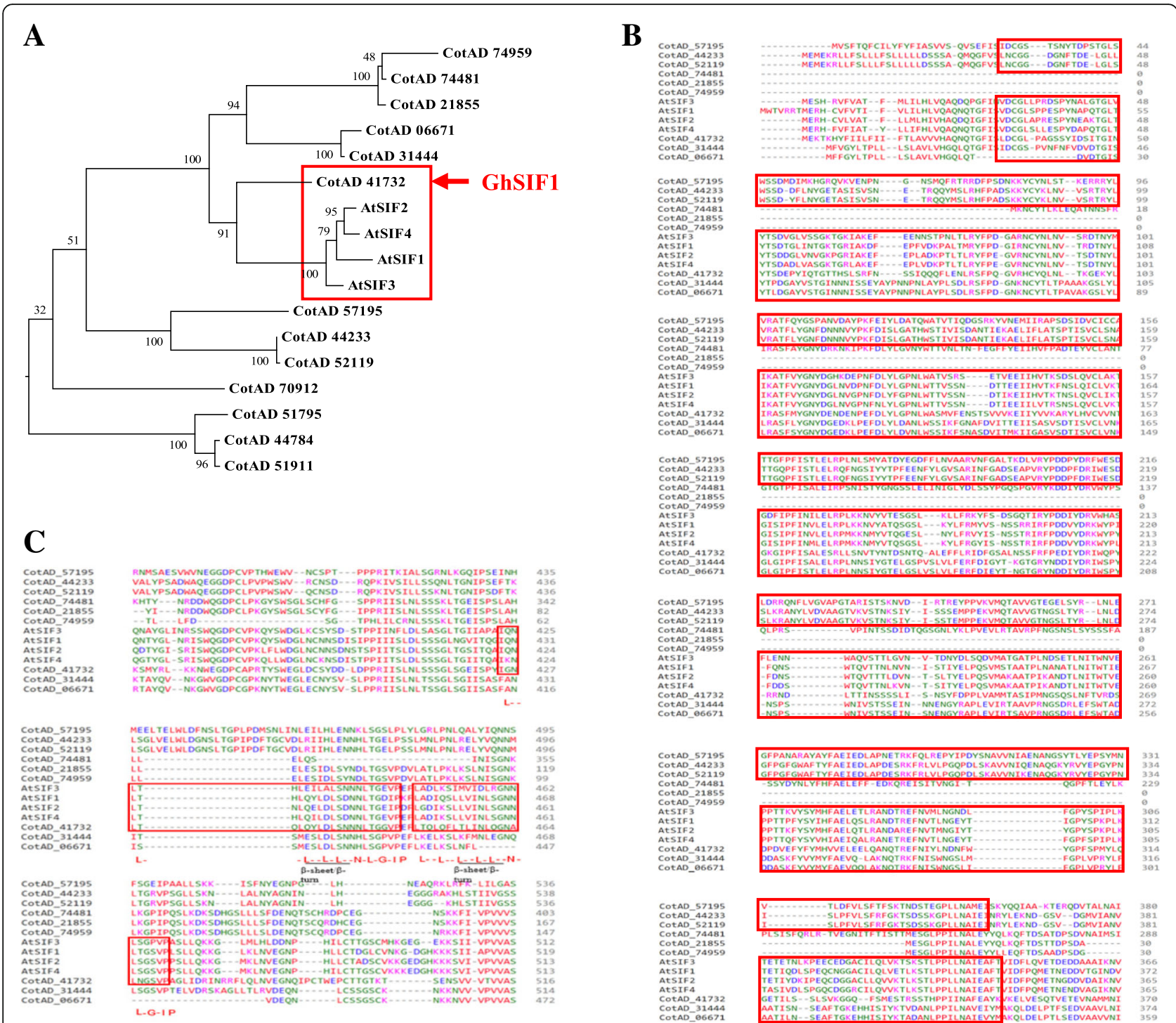

Fig. 2 Phylogenetic tree of Arabidopsis thaliana SIF family and G. hirsutum LRR-RLK subclade I protein kinases. a The phylogenetic tree is constructed using the Maximum Likelihood method based on the JTT matrix-based model with MEGA 6. The analysis involved 13 G. hirsutum LRR-RLK subclade I protein sequences with 4 of Arabidopsis thaliana SIF family protein sequences. All positions containing gaps and missing data were eliminated. b Alignment of Malectin-like domain and (c) LRR domain of AtSIFs and GhLRR-RLKs protein sequences. Protein alignment analysis was conducted with Clustal Omega (https://www.ebi.ac.uk/Tools/msa/clustalo/). In the alignment, amino acid residues are depicted with different colors for distinguishing. Ellipses represent amino acid gaps. The numbers indicate the positions of amino acid residues. Malectin-like domain in (b) and LRR domains in (c) are highlighted with red boxes. In (c), 'L--L--L-L-L--N-L--G-IP-' indicates the conserved amino acid sequence of LRR domain, and the predicted $\beta$-strand/ $\beta$-turn structure is underlined as --L-L--, where the '-' stands for non-conserved amino acid residues, the 'L' represents Leu or lle, and the 'I' represents Val or lle

with an average length of $\sim 855.8$ aa and an average molecular weight of $94.2 \mathrm{kDa}$. The CotAD_60784 protein in subclade XII was the smallest GhLRR-RLK with a length of 234 aa, while the longest protein was CotAD_44505 with a length of 1878 aa. The isoelectric point (pI) range of GhLRR-RLKs was 4.88-9.62 (Table 2 and Additional file 1: Table S1). The protein of specific interest, GhSIF1 comprised of 874 aa with a molecular weight of $98.2 \mathrm{kDa}$ and pI 5.07.
To investigate the protein structure, each GhLRR-RLKs was subjected to Blast2GO server for InterProScan domain distribution analysis [40] (Additional file 3: Figure S4 \& Additional file 1: Table S3). According to the result of InterProScan analysis, LRR and protein kinase-like domain (KD) were the two most conserved domains among the 543 GhLRR-RLK proteins, while KD was less conserved when compared to the LRR domain as it was absent in CotAD_01838 which was an outlier in the phylogenetic tree 


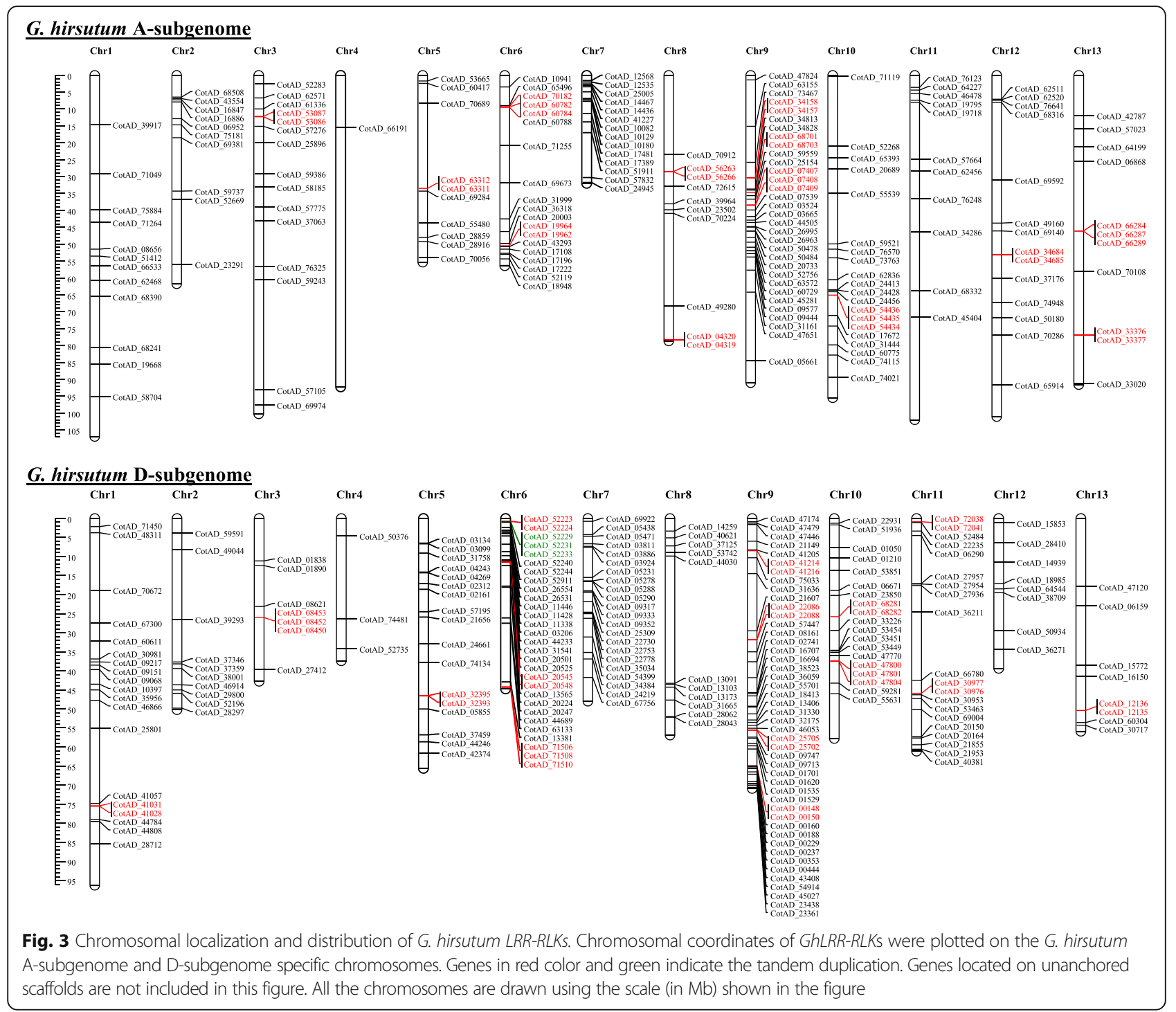

(Additional file 3: Figure S4). A Malectin-like domain was identified in 13 GhLRR-RLKs, including GhSIF1 (Additional file 3: Figure S4). Other protein domains, such as Cyclic nucleotide-binding domain (IPR000595), P-loop containing nucleoside triphosphate hydrolase (IPR027417), Kinesin motor domain (IPR001752), Glycoside hydrolase superfamily (IPR017853), Rho GDP-dissociation inhibitor domain (IPR024792), Galactose-binding domain-like (IPR008979), Gnk2-homologous domain (IPR002902), Ubiquitin domain (IPR000626), Ubiquitin-related domain (IPR029071), and Chlorophyll a/b binding protein domain (IPR023329) were also identified in some GhLRR-RLK sequences, indicating that GhLRR_RLKs may be involved in diverse functions such as protein binding, kinesin, glycoside hydrolase, ubiquitin-related, or light reception (Additional file 3: Figure S4 \& Additional file 1: Table S3).

Motif analysis using Motif Alignment \& Search Tool (http://meme-suite.org/tools/mast) with extracellular regions revealed the occurrence of 8 LRR submotifs (LRR_1, LRRNT_2, LRR_3, LRR_4, LRR_5, LRR_6, LRR_8, and LRR_9) in the LRR clan (CL0022), together with Malectin-like domain in the 13 subclades (Additional file 3: Figure S5 A-K) [41], but the distribution of these domains was highly divergent. LRR_1 and LRR_8 domains were the most abundant and were identified in $96.3 \%$ and $71.0 \%$ sequences, respectively. On the contrary, LRR_3 and LRR_5 were the rarest, which were identified in only $3.8 \%$ and 3.3\% GhLRR-RLKs, respectively. Further, a significant number (69.2\%) of subclade I members possess a Malectin-like domain in place of LRRNT_2 at the N-terminus. Interestingly, the N-terminal Malectin-like domain could only be found in subclade I, implying more special functions of the members in this clade than those of any other subclades. Although Malectin-like domain was also identified in four LRR-RLKs belonging to other Subclades (III, VIII-2, and XI-4), however they are located on the C-terminal not the 
Table 2 Molecular properties of cotton LRR-RLK gene family subclades

\begin{tabular}{|c|c|c|c|c|c|c|}
\hline \multirow[b]{2}{*}{ Subclades } & \multirow[b]{2}{*}{ No. of Genes } & \multirow[b]{2}{*}{ Gene size (Kb) } & \multirow[b]{2}{*}{ Protein size (aa) } & \multicolumn{3}{|c|}{ Range (Group-wise) } \\
\hline & & & & $\mathrm{pl}$ & Mol. Wt. (Kda) & No. of LRRs \\
\hline I & 13 & $1.674-8.986$ & 268-932 & $5.07-7.01$ & $29.1-104.3$ & 1 \\
\hline$\|$ & 28 & $2.911-7.585$ & $491-650$ & $5.24-8.51$ & $54.7-71.7$ & $1-2$ \\
\hline III & 89 & $1.793-7.562$ & $343-1067$ & $5.56-9.46$ & $37.4-115.7$ & $1-6$ \\
\hline IV_1 & 5 & $2.696-3.355$ & $865-974$ & $5.5-7.02$ & $94.6-107.5$ & $3-8$ \\
\hline IV_2 & 7 & $2.156-2.580$ & $640-684$ & $6.44-8.61$ & $69.9-75.6$ & 1 \\
\hline V & 18 & $3.730-7.210$ & $690-835$ & $5.56-8.79$ & $76.1-91.1$ & $1-3$ \\
\hline VI_1 & 7 & $2.204-2.558$ & $680-852$ & $6.3-9.47$ & $75.6-93.8$ & $1-6$ \\
\hline VI_2 & 23 & $2.642-6.460$ & $573-790$ & $4.88-9.11$ & $63.6-88.0$ & $1-3$ \\
\hline VII_1 & 10 & $2.745-3.425$ & $885-1141$ & $5.44-6.94$ & $96.0-124.8$ & $1-8$ \\
\hline VIII_1 & 31 & $3.565-13.805$ & $661-1097$ & $5.04-8.00$ & $72.9-121.1$ & $1-4$ \\
\hline VIII_2 & 9 & $4.463-12.060$ & 819-1343 & $5.49-8.68$ & $88.4-147.2$ & $1-3$ \\
\hline IX & 18 & $2.840-3.845$ & 893-979 & $5.38-8.83$ & $98.1-107.0$ & $1-3$ \\
\hline X_1 & 3 & $3.123-3.585$ & 958-1099 & $5.46-7.03$ & $105.9-121.0$ & $3-5$ \\
\hline X_2 & 9 & $2.762-3.859$ & $920-1140$ & $6.03-8.43$ & $100.5-124.7$ & $1-7$ \\
\hline X_3 & 24 & $1.577-3.842$ & $525-1280$ & $5.7-7.85$ & $58.1-139.9$ & $1-6$ \\
\hline X_4 & 7 & $2.745-2.939$ & 885-944 & $5.71-8.91$ & $97.3-102.8$ & $2-4$ \\
\hline$X I \_1$ & 76 & $1.942-9.841$ & 408-1878 & $5.16-9.13$ & $45.5-207.6$ & $1-5$ \\
\hline$X I \_2$ & 3 & $1.919-2007$ & $639-643$ & $8.82-9.08$ & $71.1-71.3$ & 2 \\
\hline$X I \_3$ & 4 & 2.339-2.918 & 556-945 & $5.89-8.13$ & $60.8-104.2$ & $1-4$ \\
\hline$X I \_4$ & 10 & 3.049-4.399 & 808-974 & $6.73-8.89$ & 88.9-99.9 & $2-4$ \\
\hline XII_1 & 77 & $1.811-12.435$ & $441-1539$ & $5.53-9.02$ & $48.1-170.0$ & $1-7$ \\
\hline$X I I \_2$ & 51 & $1.259-13.013$ & 234-1209 & $4.96-9.62$ & $26.0-133.5$ & $1-7$ \\
\hline$X I I I$ & 14 & $3.211-6.506$ & $400-1829$ & $5.51-8.23$ & 43.7-198.9 & $1-7$ \\
\hline Others & 1 & 3.003 & 948 & 5.54 & 104.9 & 5 \\
\hline
\end{tabular}

N-terminal of the protein. A total of 391 GhLRR-RLKs consisted of various signal peptides at their N-terminal (Additional file 3: Figure S6 \& Additional file 1: Table S4), however each GhLRR-RLK comprised a transmembrane domain (Additional file 1: Table S4). The protein structure analysis showed that GhSIF1 consisted of a 22-aa signal peptide, a Malectin-like domain, an LRR-8 motif, a transmembrane domain, and an intracellular kinase domain (Additional file 3: Figure S5 A and Additional file 1: Table S4).

\section{Functional annotation and gene ontology analysis}

Cellular component analysis conducted with Blast2GO software showed that 542 GhLRR-RLKs were predicted to be located on the membrane system, and 538 proteins were predicted to be localized in cell part, followed by organelle (286), membrane part (209), symplast (206), and cell junction (206) (Additional file 3: Figure S7 and Additional file 1: Table S3) while some proteins were predicted to be extracellular (95). The biological processes analysis (Additional file 3: Figure S7 and Additional file 1: Table S3) showed that the GhLRR-RLKs are involved in 'cellular process' (504), 'response to stimulus' (502), 'single-organism process' (498), 'biological regulation' (476), 'signaling' (411), and 'metabolic process' (410). Some proteins obtained the GO terms 'developmental process' (335), 'multicellular organismal process' (321), 'reproduction' (261), which were followed by 'multi-organism process' (168), 'cellular component organization or biogenesis' (146), and 'localization' (109). Molecular function analysis showed most GhLRR-RLKs displayed 'catalytic activity' (517), 'binding' (513), 'signal transducer activity' (152) and 'molecular transducer activity' (127) functions (Additional file 3: Figure S7 and Additional file 1: Table S3). A detailed information on specific cellular component, biological processes, and molecular function was performed and presented in the additional information (Additional file 3: Figure S8-S10). Specifically, GhSIF1 was predicted to be a negative regulation factor of an abscisic acid-activated signaling pathway, indicating it may play a negative role in the abiotic stress 


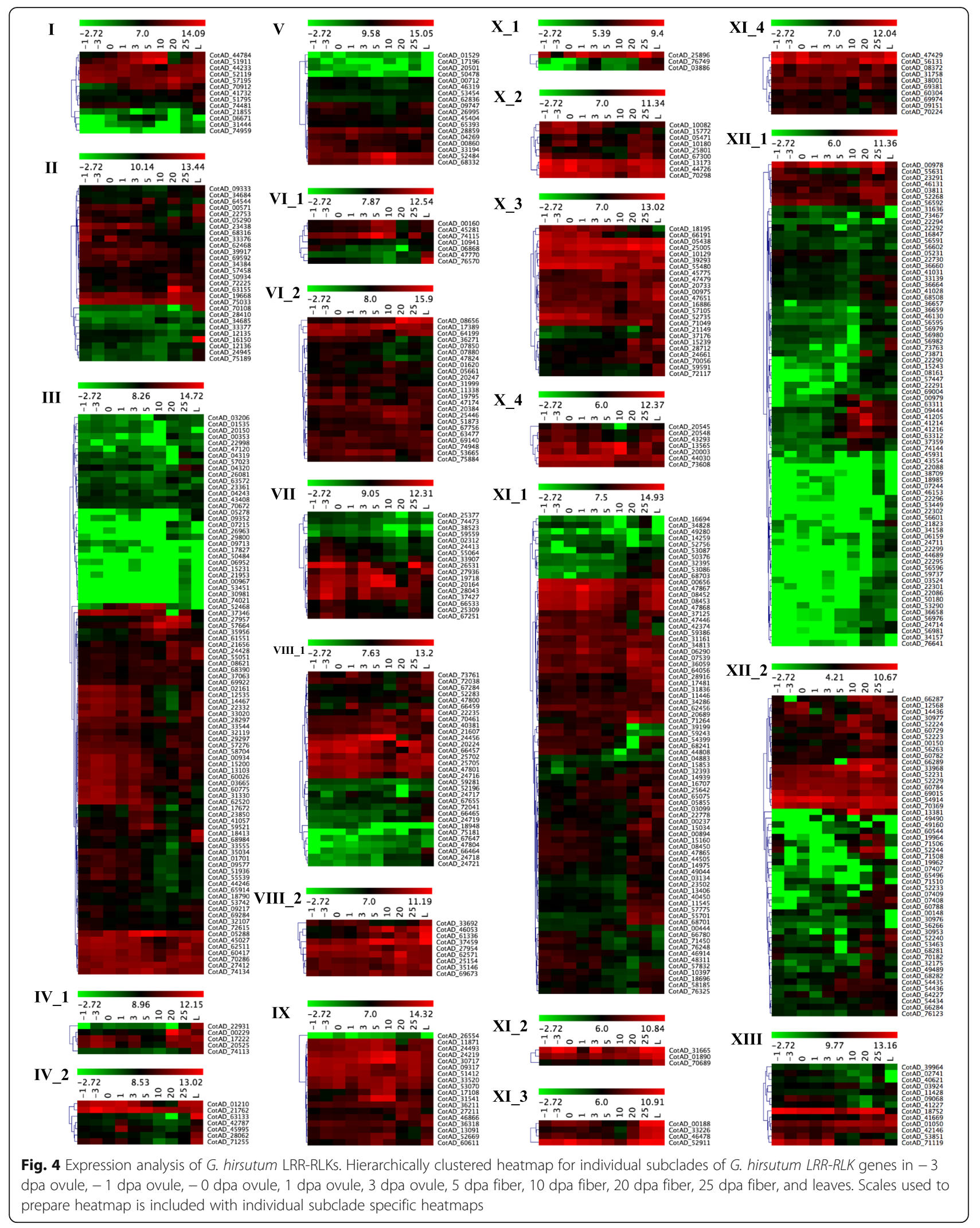




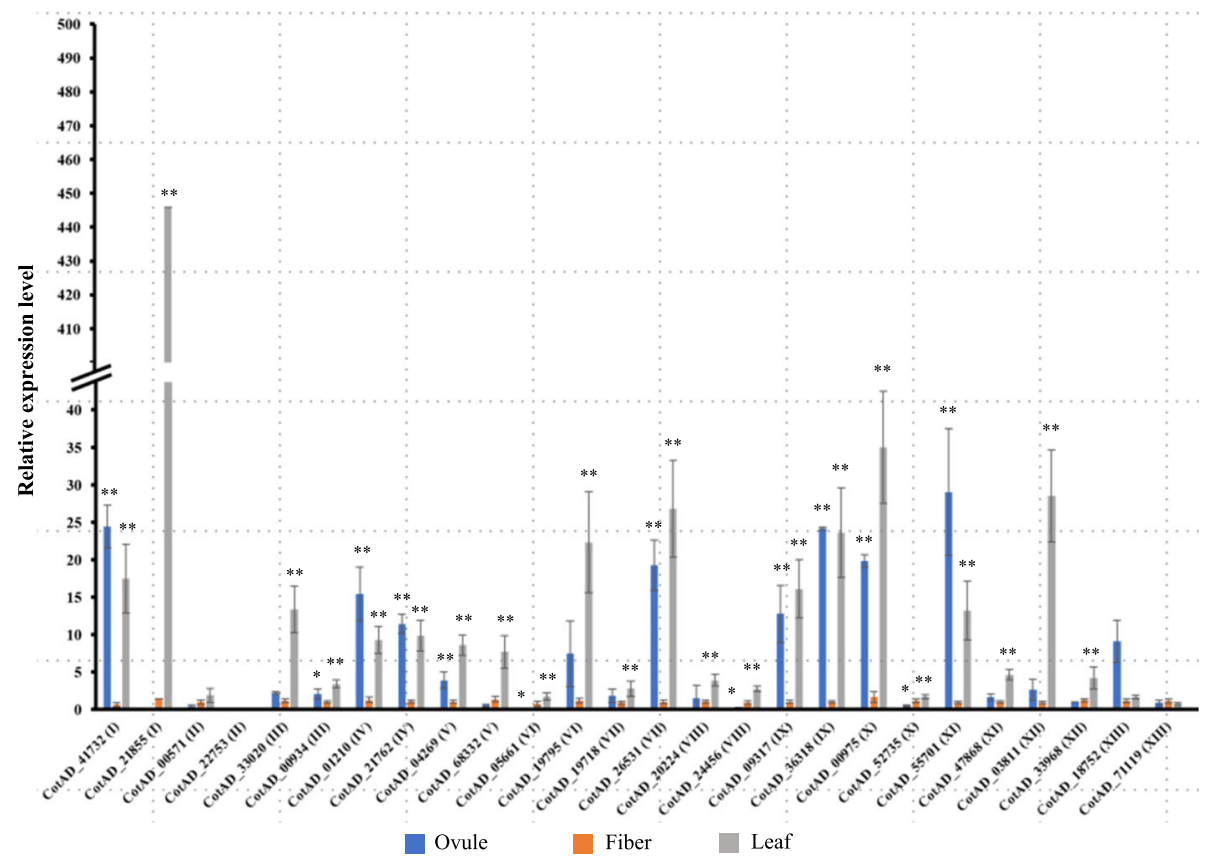

Fig. 5 Real-time RT-PCR analysis of G. hirsutum LRR-RLKs expression. Ovule, fiber, and leaf tissue samples were collected at 5 dpa from cotton plants grown in the green house for real-time RT-PCR analysis. The expressions of $26 \mathrm{G}$. hirsutum LRR-RLKs in various subclades were analyzed. GhActin2 was used as the internal reference gene. Data shown are an average of three technical replicates for three independent biological replicates. Error bars represent S.D. $(n=9)$. The statistically significant difference between fiber and other tissues was determined by t-test. $P<0.05$ was marked as *. $P<0.01$ was marked as **

tolerance mechanism (Additional file 1: Table S3). Furthermore, the Blast2Go also indicated that GhSIF1 could even respond to biotic stress (Additional file 1: Table S3).

\section{GhLRR-RLK gene expression analysis in various organs and across fiber developmental stages}

Publicly available cotton transcriptome datasets from $G$. hirsutum TM-1 were used to investigate the expression pattern of 543 LRR-RLK genes in leaves and across the different fiber developmental stages $(-3,-1,0,1,3,5,10$, 20, and 25 dpa (day post anthesis)) (Fig. 4 and Additional file 1: Table S5 and S6). Subclade specific heatmaps were generated to show the expression pattern of LRR-RLK genes using the self-normalized log converted RPKM values obtained by mapping transcriptome datasets (Additional file 3: Figure S11). Most of the genes of subclades VI_2, VIII_2, IX, X_2, X_3, X_4, XI_2, XI_3 and XI_4 showed higher expression in all the stages of cotton fiber development indicating a potential role of these subclades genes in fiber development. However, members of I, II, III, IV_1, IV_2, V, VI_1, VII, VIII_1, X_1, XI_1, XII_2, and XIII subclades showed clusters of genes with low, moderate as well as high expression levels at various stages of fiber development. Most of the genes belonging to cluster XII_1 were low to moderately expressed except one small sub-cluster of highly expressed genes.

To further confirm the expression of $L R R-R L K$ genes, quantitative PCR analysis was performed with 26 GhLRR-
$R L K$ genes (two representative genes from each subclade) including GhSIF1 (CotAD_41732) in leaf, 5 dpa ovule and 5 dpa fibers. As shown in Fig. 5, most of the GhLRR-RLK genes exhibited similar expression patterns as they had a significantly higher expression in ovule and leaf tissues than that in fiber tissue, except CotAD_00571, CotAD_52735 and CotAD_71119, which were expressed at similar levels in all three tissues. Specifically, CotAD_22753 could not be detected in any tissues, consistent with the transcriptome results.

\section{Gene expression and transient silencing of AtSIF homolog in cotton}

The real-time PCR result showed that GhSIF1 was significantly down-regulated in the salt-treated root tissue (Fig. 6a), similar to Arabidopsis SIF1 and SIF2 indicating a potential role of GhSIF1 in the salt tolerance in cotton [32]. To further study the function of GhSIF1, we transiently silenced GhSIF1 expression in cotton plants using Tobacco Rattle Virus (TRV) mediated virus-induced gene silencing system [42]. A 371 bp GhSIF1 cDNA fragment was inserted in the TRV-2 to transiently silence GhSIF1 mRNA using agroinfiltration. The region was selected from the specific 3'UTR (Untranslated Region) as the coding region showed high homology among LRR-RLKs. Ten days old cotton plants with two cotyledon leaves were infiltrated with pTRV1 and with pTRV2 (GhSIF1) 
A

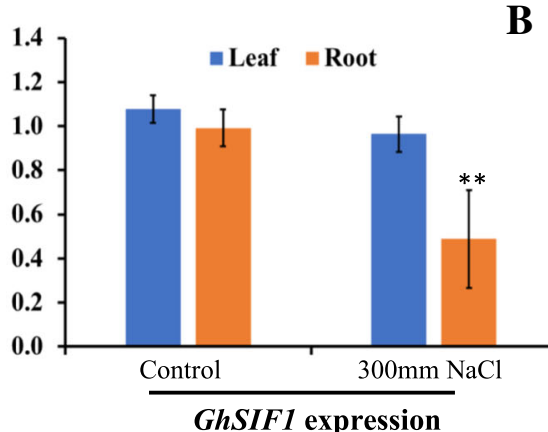

GhSIF1 expression

B

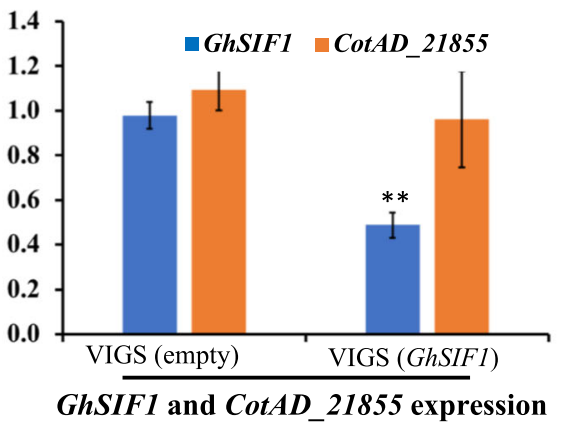

C

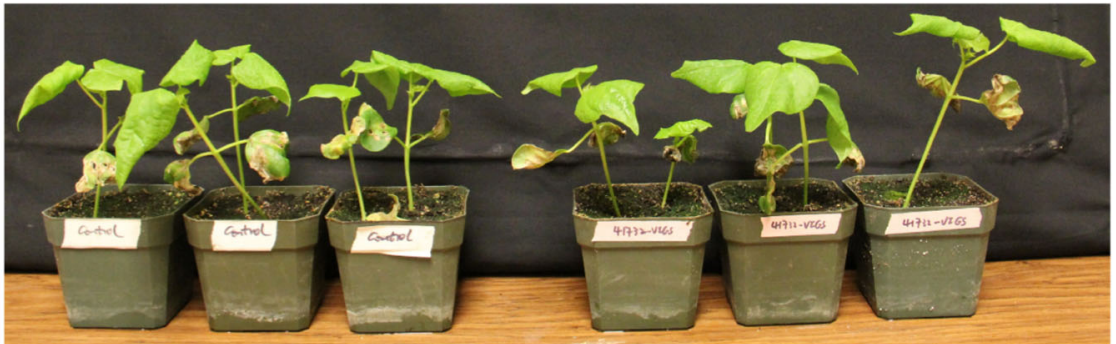

D

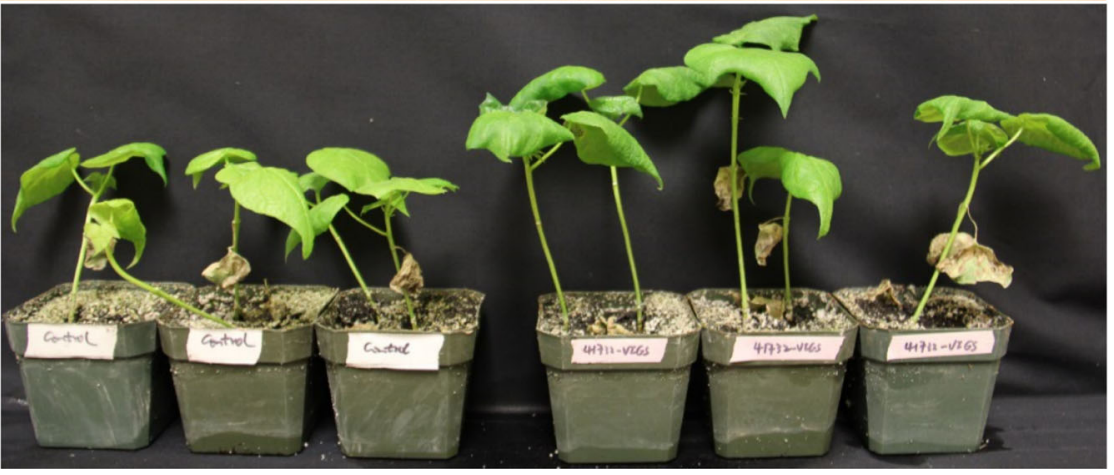

VIGS (empty)

VIGS $(G h S I F 1)$

Fig. 6 Expression and phenotypic analyses of GhSIF1 under salt treatment and in VIGS treat cotton plants. (a) Cotton (G. hirsutum) seeds germinated on 1/2 MS medium were transferred to $1 / 2$ MS with or without $300 \mathrm{mM} \mathrm{NaCl}$ medium. Ten days later, leaves and roots were collected for real-time PCR analysis. GhActin2 was used as the reference gene. (b) 10 days old cotton plants (G. hirsutum) with two cotyledon leaves were infiltrated with TRV1 and empty TRV2 (as control) or TRV2-GhSIF1 (targeting GhSIF1 mRNA). Ten days later, leaf samples were collected for real-time PCR analysis. GhActin2 was used as the reference gene. Data shown are an average of three technical replicates for two independent biological replicates. Error bars represent S.D. $(n=6)$. The statistically significant difference was determined by t-test. $P<0.05$ was marked as *. $P<0.01$ was marked as **. Pictures were taken (c) before salt treatment and (d) 18 days after salt treatment

along with pTRV1 and pTRV2 (empty) as a control. Leaf samples of control as well as GhSIF1 targeting plants were collected 10 days after infiltration for gene expression analysis. The expression of GhSIF1 was significantly down-regulated in VIGS (GhSIF1) infiltrated plants compared to the control plants (Fig. 6b). To insure the specificity of VIGS mediated suppression of GhSIF1, the expression of another gene CotAD_21855, which has $66 \%$ similarity with GhSIF1 CDS (Coding Sequence) was analyzed. Gene expression analysis showed that the expression of CotAD_21855 was not affected in the pTRV2(GhSIF1) silenced plant indicating the specificity of the VIGS system towards GhSIF1 (Fig. 6b).
Evaluation of salt tolerance of the GhSIF1 silenced plants Gene silenced plants were evaluated for the salt tolerance in the presence of $300 \mathrm{mM} \mathrm{NaCl}$ for 2 weeks. Cotton plants with GhSIF1 silencing exhibited better performance compared to control plants (Fig. 6c \& d). The results showed that GhSIF1 silenced plants displayed significantly longer shoot and more biomass than the control plants (Fig. 7). Previous studies showed that salt stress induce the reactive oxygen species, which results in chlorophyll degradation and membrane permeability leading to the reduction in chlorophyll content and high electrolyte leakage [43, 44]. The results showed that the chlorophyll content was significantly higher, while the electrolyte leakage was much lower in GhSIF1 


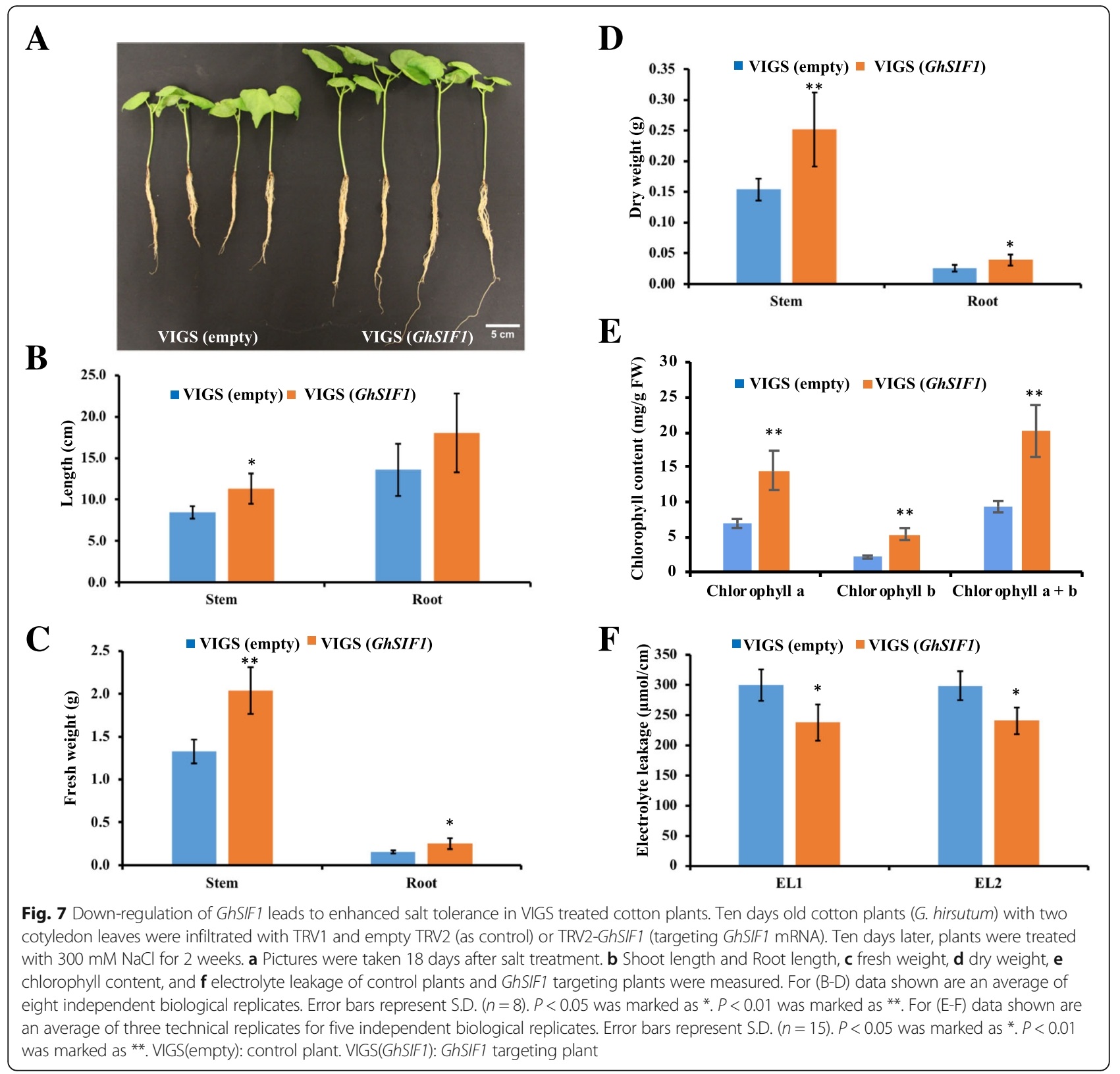

silenced plants than in control plants (Fig. 7e \& f), indicating that knock-down of GhSIF1 gene in cotton resulted in increased salt tolerance.

\section{Discussion}

In plants, LRR-RLKs are one of the most important membrane-anchored receptors, which transduce the apoplastic signals into symplast and then trigger the downstream responses. Various studies have shown that LRR-RLKs involve in many fundamental biological processes in plants, such as phytohormone perception, plant development, and responses to the adverse environment [14-19]. The presence of large numbers in the LRR-RLK gene family makes the functional characterization of individual member difficult due to functional redundancy. Arabidopsis offers an excellent model for functional characterization of LRR-RLK genes due to their relatively fewer numbers and the availability of genetic and genomic resources. We have previously identified and characterized Arabidopsis SIF2, a negative regulator of salt tolerance [32]. The present investigation identified a homolog of AtSIF gene in cotton by phylogenetic analysis and functionally characterized for its role in salt tolerance using transient gene silencing system.

\section{Cotton LRR-RLK gene family constitutes one of the biggest gene families in the plant kingdom}

Due to their diverse and critical roles in signal transduction, plant development, photomorphogenesis, and abiotic/biotic 
stress responses, LRR-RLKs constitute one of the largest gene families in the plant and animal kingdoms. The present study identified 543 LRR-RLK genes and the number is much larger than that of diploid plant species Arabidopsis (234) and rice (309). It is also larger than paleopolyploid soybean (467) and allohexaploid wheat $(531)[12,24,28,30]$. This high number of genes is likely due to cotton's complex allotetraploid genome and long evolutionary history along with complex traits such as specialized fibers. In addition to the complex genome, cotton produces longest single cell in the plant kingdom composed of $\sim 96 \%$ cellulose which requires precise developmental regulation.

Cultivated cotton (G. hirsutum) is an allotetraploid organism which is the result of the hybridization of two diploid progenitor relatives G. arboreum (AA) and G. raimondii (DD) [45]. Each of the two progenitors provided one set of 13 chromosomes to G. hirsutum leading to genome doubling in the cultivated G. hirsutum $\left(\mathrm{A}_{t} \mathrm{~A}_{t} \mathrm{D}_{t} \mathrm{D}_{t} ; 2 n=4 \times=52\right)$ [46]. Analysis of chromosomal location provides the information about the position of a gene on the specific chromosome. However, it does not provide information about the nature of its origin, hence we performed gene duplication analysis. Chromosomal distribution analysis showed that the distribution patterns of $L R R-R L K$ genes on A-subgenome and D-subgenome were very similar (Fig. 3 \& Additional file 3: Figure S1) in terms of the number and location. Nevertheless, the numbers of LRR-RLKs on A- and D-subgenomes are not equal, as A-subgenome carries 179 genes while D-subgenome carries 219 genes, which could be due to independent evolution of the parental diploid species before hybridization to form tetraploid species.

\section{The diversity of LRR-RLKs protein structure and functional significance}

The exon-intron structure analysis showed a conservative pattern among the subclades while, the protein motif analysis revealed that protein members within the same subclade showed similar motifs, localization pattern and potentially similar functions (Additional file 3: Figure S2 A-I \& Additional file 3: Figure S5 A-K). For instance, the extracellular Malectin-like domain (IPR024788) helps in recognition of and binding to Glc-N-glycan of Endoplasmic Reticulum [47]. In Arabidopsis, all the LRR-RLKs having N-terminal Malectin-like domain were grouped in subclade I, and several of them have been proved to be involved in biotic stress resistance [48, 49]. The extracellular $\mathrm{N}$-terminal Malectin-like domain is a complex structure offering proteins the ability to recognize and bind Glc-N-glycan of Endoplasmic Reticulum, and several Arabidopsis LRR-RLK proteins containing this domain have been proved to be involved in biotic stress resistance [47-49]. Similarly, in cotton, N-terminal Malectin-like domain was identified in 9 LRR-RLKs, and all of them were grouped in subclade I in the phylogenic analysis. Due to the diverse functional roles of LRR-RLK proteins, these proteins have specialized domains for functional specializations. For instance, the extracellular LRR domain allows RLK to perceive a specific ligand, and the transmembrane domain allows it to firmly anchor on the plasma membrane, while the protein kinase-like domain offers its phosphorylation ability allowing it to transduce the signal to downstream signaling pathway. In the presence of a bacterial pathogen, the LRR domain of Arabidopsis BAK1 will instantly form a complex with the LRR domain of another LRR-RLK protein FLS2 [13]. The conformational change caused by this extracellular complex will activate the kinase domain of BAK1 to autophosphorylate itself and then transphosphorylate kinase domain of FLS2, followed by the activation of downstream signaling cascades [50].

\section{LRR-RLKs are involved in multiple biological processes in cotton}

LRR-RLK gene family is a multigene family involved in various functions in cotton, however, only a very few GhLRR-RLK genes have been functionally characterized [34-37]. Biological process analysis indicated that GhLRR$R L K \mathrm{~s}$ have multiple molecular functions such as response to stimulus (502), biological regulation (476), signaling (411), metabolic process (410), developmental process (335) and reproduction (261), which underline their potential functions in plant development, environmental stress, metabolism and reproduction through signal transduction mechanism (Additional file 3: Figure S7). In addition, cotton is unique in producing highly specialized single cells called cotton fibers from the seed coat epidermal cells. These cells follow a unique developmental pattern with primary and secondary cell wall deposition leading to the deposition of $\sim 96 \%$ cellulose. LRR-RLKs have been shown to be involved in the cotton fiber development as well as cell wall biosynthesis in cotton. GhRLK1 was induced in developing cotton fibers and was predicted to be involved in the secondary cell wall synthesis in cotton fiber [34]. The RNAseq analysis of publicly available dataset (Fig. 4 \& Additional file 3: Figure S11) showed that LRR-RLKs genes belonging to subclades VI_2, VIII_2, IX, X_2, X_3, X_4, XI_2, XI_3 and XI_4 are highly abundant across most of the fiber developmental stages while genes belonging to the subclades I, II, III, IV_1, IV_2, V, VI_1, VII, VIII_1, X_1, XI_1, XII_2 and XIII showed variable expression pattern. Further, the real-time RT-PCR expression analysis of 26 genes in leaf, $5 \mathrm{dpa}$ fiber and $5 \mathrm{dpa}$ ovule suggested that most of these genes were expressed in all three tissues, however, expression was significantly higher in leaves followed by ovules (Fig. 5). Out of the 26 genes, CotAD_22753 was not detectable in any of these three tissue types whereas CotAD_00571, CotAD_52735, and 
CotAD_71119 exhibited consistent expression across the three tissues (Fig. 5).

\section{GhSIF1 is a negative regulator of salt tolerance in cotton} Due to presence of a large number of genes in the LRR-RLK gene family and functional redundancy, the complete understanding of their role in plant growth, development and stress responses are lagging behind. In Arabidopsis, only 35 genes have been functionally characterized [12] which indicates the complexity involved in the functional characterization of the LRR-RLK family genes. Functional analysis of these genes in tetraploid cotton with a much bigger gene family and long life cycles coupled with transformation hindrances, it will be difficult to completely characterize all the GhLRR-RLK genes in cotton. The present study provides a comprehensive analysis of cotton LRR-RLKs, which will help in rapid identification and characterization of cotton genes using translational research and advanced functional genomics tools. Particularly, with the information from the characterized Arabidopsis genes, it is possible to predict and functionally characterize the respective cotton homologous gene. We have recently identified a subfamily of AtLRR-RLK gene family (SIF gene family; SIF1-SIF4), which is shown to be involved in both biotic and abiotic stress responses. Particularly, knocking out of SIF1 and SIF2 significantly enhanced the salt tolerance of Arabidopsis [32]. Interestingly, the phylogenetic analysis using Arabidopsis SIF gene family showed that only one gene, GhSIF1 has a very close evolutionary relationship with AtSIFs (Fig. 2). By generating highly specific VIGS construct targeting GhSIF1, we have functionally characterized its role in salt tolerance in cotton paving the way for rapid functional characterization of cotton genes using translational research. The transiently silenced cotton plants showed enhanced salt tolerance, indicating that GhSIF1, similar to AtSIFs in Arabidopsis, is a negative regulator of plant salt tolerance (Figs. 5, 6 and 7). The transient characterization is highly practical for rapid functional characterization of genes due to the recalcitrance, laborious and time-consuming stable transformation in cotton.

\section{Conclusions}

The present investigation performed a genome-wide analysis of LRR-RLK family genes in cultivated tetraploid cotton G. hirsutum leading to the identification of 543 GhLRR-RLKs. Five hundred forty-two GhLRRRLKs were grouped into 13 subclades while remaining one protein, CotAD_01838, was not assigned to any subclade. These GhLRR-RLK genes were distributed on all 13 chromosomes of both A and D subgenomes but at a different frequency, and a total of 42 tandem duplication events were identified involving 110 genes. Our results also indicated that each LRR-RLKs subclade has distinctive gene structure and the protein structure. Gene expression analysis and functional annotation indicated that GhLRR-RLKs were spatiotemporally expressed and potentially involved in various biological processes in different tissues or cell types. Genome-wide analysis and phylogenetic analysis led to the identification of six Arabidopsis SIF homologs in cotton. Among them, GhSIF1 has the highest conserved amino acid sequence with AtSIF subfamily. Functional studies demonstrated that the salt tolerance function of GhSIF1 is conserved with AtSIF1 and AtSIF2. This offers an excellent opportunity to silence the GhSIF1 to develop salt-tolerant cotton using genome editing technologies as GhSIF1 is a negative regulator of salt tolerance.

\section{Methods}

Identification of LRR-RLK gene family in G. hirsutum

For the in-silico identification of LRR-RLK gene family in upland cotton, G. hirsutum reference genome data was downloaded from the CottonGen database (https:// www.cottongen.org/data/download/genome) [46, 51]. Arabidopsis LRR-RLK family 234 gene ids were pooled from the previous reports and their protein sequences were retrieved from TAIR10 database (https://www.arabidopsis.org/) [11, 12, 52]. A BlastP similarity search (https://blast.ncbi.nlm.nih.gov/Blast.cgi?CMD=Web\&PAGE_TYPE=BlastDocs\&DOC_TYPE=Download) was performed against the G. hirsutum reference proteome data using Arabidopsis LRR-RLK family protein sequences as the query at default parameters with an e-value of $10^{-10}$. Non-redundant protein sequences obtained from BlastP search were analyzed for the presence of Leucine-Rich Repeats (LRRs) and kinase domain using the online hmmscan search tool (HMMER; https://www.ebi.ac.uk/Tools/ hmmer/search/hmmscan) [53] and NCBI's Conserved Domains Database (CDD; http://www.ncbi.nlm.nih.gov/ Structure/cdd/wrpsb.cgi) [54]. Further, proteins with minimum of one LRR repeat and a kinase domain were analyzed for the presence of transmembrane helices using online available TMHMM server v.2.0 (http:// www.cbs.dtu.dk/services/TMHMM/) [55]. Upland cotton protein sequences with minimum of 1 LRR repeat, kinase domain and transmembrane helices were classified as GhLRR-RLK gene family members and were used for further characterization. For the identified GhLRR-RLK genes, we continued to use the original gene id provided in the reference genome [46].

\section{Phylogenetic analysis of GhLRR-RLK proteins}

To further classify into subclades based on their sequence similarity with Arabidopsis LRR-RLK proteins, phylogenetic analysis of GhLRR-RLK family members was performed using Molecular Evolutionary Genetics 
Analysis (MEGA) v6.06. LRR-RLK proteins from cotton (543) and Arabidopsis (234) were subjected to multiple alignment using ClustalW sequence alignment program of MEGA v6.06 [56] with default parameters. Further, a phylogenetic tree was constructed with MEGA v6.06 using Neighbor-Joining (NJ) method. Bootstrap replicates of 1000 along with other default parameters (phylogenetic reconstruction, substitution type: amino acids, model/ methods: p-distance, rates among sites: uniform rates and gap missing data treatment: partial deletion) were used to create the phylogenetic tree. Based on the presence of previously classified Arabidopsis LRR-RLK proteins, branches were classified into 23 LRR-RLK sub-groups. Phylogenetic analysis of AtSIF family and GhLRR-RLK subclade I was performed on the phylogeny.fr server (www.phylogeny.fr) [57].

\section{Physical properties, gene structure and chromosomal localization analysis}

The identified cotton $L R R-R L K$ genes were grouped into subclades and analyzed further for detailed characterization. Gene length, protein size, location and orientation on the chromosomes were retrieved from the reference genome dataset. Other physical properties such as theoretical pI and molecular weight of the LRR-RLK proteins were calculated using the ExPASy server's Compute $\mathrm{pI} / \mathrm{Mw}$ tool (http://web.expasy.org/compute_pi/). For the chromosomal localization analysis, chromosomal coordinates of the cotton $L R R-R L K$ genes were plotted on the G. hirsutum Aand D-subgenome specific chromosomes separately using the Mapchart 2.30 software. For the gene structure analysis, exon-intron coordinates for each GhLRR-RLK genes were fetched from the .gff file and diagrammatically represented using the Gene Structure Display Server 2.0 [58].

\section{Tandem duplication among cotton LRR-RLK genes}

Tandem duplication among the $L R R-R L K$ gene family was analyzed by comparing their position on the chromosome/ scaffold. Adjacent genes with a maximum of one gene interruption were considered as tandemly duplicated genes. In some cases, adjacent genes interrupted by a maximum of two genes were also considered tandemly duplicated if they were within $1 \mathrm{MB}$ region.

\section{Protein structure analysis, domains distribution, and annotation analysis}

InterProScan domains and Blast2GO annotation analysis were conduct with 543 G. hirsutum LRR-RLK protein sequences using Blast2GO tool suite according to the software instruction [40]. The extracellular structure of GhLRR-RLK proteins was analyzed with Motif Alignment \& Search Tool on Motif-based sequence analysis online tools [59]. Reference motifs (LRR clade domains and Malectin-like domain) were obtained from the NCBI's
Conserved Protein Domain database (https:// www.ncbi.nlm.nih.gov/Structure/index.shtml). Signal peptide identification was performed on the SignalP 4.1 Server (http://www.cbs.dtu.dk/services/SignalP/) [60]. Transmembrane domain analysis was performed using the TMHMMserver V.20 on SignalP 4.1 Server.

\section{In-silico gene expression analysis of GhLRR-RLKs}

Transcriptome datasets were obtained from NCBI's Short Read Archive (SRA) database (http://www.ncbi. nlm.nih.gov/sra) for different cotton fiber developmental stages $(-3,-1,0,1,3,5,10,20$, and $25 \mathrm{dpa})$ and leaves from G. hirsutum TM-1 (Additional file 1: Table S5). Reads from different datasets were mapped on GhLRR-RLK family related genes using the QSeq program of DNASTAR Lasergene package (http://www.dnastar.com/t-nextgen-qseq.aspx). Hierarchically clustered heatmaps for individual sub-groups were created with the $\mathrm{MeV}$ (http://mev.t$\mathrm{m} 4$.org/\#/welcome) using the self-normalized log converted RPKM (Reads per Kilobase per Million reads) values calculated by the QSeq program. Apart from this, another heatmap showing the expression of all the cotton $L R R-R L K$ genes was created using the QSeq heat map option.

\section{Plant growth, RNA isolation, cDNA synthesis and quantitative PCR analysis}

G. hirsutum TM-1 seeds were germinated on soil and the plants were grown under a $16 \mathrm{~h}$-light $/ 8 \mathrm{~h}$-dark photoperiod at $28{ }^{\circ} \mathrm{C}$ in the growth chamber (Percival, Perry, Iowa) and moved to the green house for maturity to produce cotton fibers. Plant total RNA was isolated with Spectrum plant total RNA kit (Sigma-Aldrich, USA) from $100 \mathrm{mg}$ plant sample according to the manufacturer's instructions. The first strand cDNA was synthesized using iScript Reverse Transcription Supermix for RT-qPCR (Bio-Rad, USA) with $1 \mu \mathrm{g}$ total RNA according to the manufacturer's instruction. Real-time PCR was performed with FastStart Essential DNA green Master (Roche, Swiss) according to the manufacturer's instructions. LightCycler 96 (Roche, Swiss) was used for the real-time PCR experiments. Real-time PCR results were calculated by using the $\Delta \Delta \mathrm{Ct}$ method [61].

\section{Plasmid construction and transient gene silencing}

For pTRV2(GhSIF1) plasmid construction, a 371 bp 3' UTR fragment of GhSIF1 cDNA was amplified from $G$. hirsutum cDNA pool with NEBNext Q5 High-Fidelity polymerase (NEB, U.S.A). The pTRV vectors were obtained from the TAIR (https://www.arabidopsis.org/abrc/ catalog/vector_3.html) [62]. The 3' UTR region on GhSIF1 was carefully selected to avoid off targeting of the VIGS system. The primers used to amplify the cDNA fragment were forward primer 5'-AAATCTAGA TCAAATCATTAAATTTGATGCCTTTC-3' with XbaI 
restriction site, and reverse primer 5'-AAAGAGCTC AATTCTTATTTACAAAAAAGCCATC-3' with SacI restriction site. The PCR product was then digested with $X b a \mathrm{I}$ and $S a c \mathrm{I}$, and sub-cloned into the binary vector pTRV2 digested with the same set of enzymes, resulting in $2 \times \mathrm{p} 35 \mathrm{~S} / \mathrm{CP} /$ GhSIF1/Rbz/nos. pTRV1, pTRV2(empty), and pTRV2(GhSIF1) plasmids were then mobilized into Agrobacterium tumefaciens strain GV3101 for virus-induced gene silencing. Virus-induced gene silencing of cotton was performed following the published protocol [63].

\section{Determination of chlorophyll content and electrolyte leakage measurements}

For determination of chlorophyll content, $300 \mathrm{mg}$ of youngest leaf samples were collected from cotton plants from the growth chambers. The leaf samples were then sliced into small pieces and ground to fine powder using liquid nitrogen, which was then transferred to $15 \mathrm{ml}$ Falcon tube with $5 \mathrm{ml}$ of $80 \%$ acetone for chlorophyll extraction. After $30 \mathrm{~min}$ of incubation under room temperature, the falcon tubes were centrifuged at $4{ }^{\circ} \mathrm{C}$ for $15 \mathrm{~min}$ at $3000 \mathrm{rpm}$, and the supernatant was then transferred to $50 \mathrm{ml}$ falcon tube with $10 \mathrm{ml} \mathrm{80 \%}$ acetone and kept in the dark until chlorophyll content was determined.

Absorbance of the extract was measured at $645 \mathrm{~nm}$ and $663 \mathrm{~nm}$ by using a spectrometer, and the chlorophyll concentrations are calculated as following equation:

$$
\begin{aligned}
& \text { Chlorophyll a content }(\mathrm{mg} / \mathrm{g})=\left(12.7 \times \mathrm{A}_{663}-2.69\right. \\
& \left.\times \mathrm{A}_{645}\right) \times \mathrm{V} / \mathrm{W} / 1000 \\
& \begin{aligned}
\text { Chlorophyll b content }(\mathrm{mg} / \mathrm{g})= & \left(22.9 \times \mathrm{A}_{645}-4.86\right. \\
\times & \left.\mathrm{A}_{663}\right) \times \mathrm{V} / \mathrm{W} / 1000 \\
\text { Chlorophyll }(\mathrm{a}+\mathrm{b}) \text { content }(\mathrm{mg} / \mathrm{g}) & =\left(8.02 \times \mathrm{A}_{663}\right. \\
& \left.-20.20 \times \mathrm{A}_{645}\right) \\
& \times \mathrm{V} / \mathrm{W} / 1000
\end{aligned}
\end{aligned}
$$

Where: $\mathrm{V}=$ volume of the extract $(\mathrm{ml}) ; \mathrm{W}=$ fresh weight of the leaf samples (mg).

For the determination of electrolyte leakage, fresh leaf disc was cut from the youngest leaf and immersed in $5 \mathrm{ml}$ of deionized water. The sample was then incubated at $32^{\circ}$ $\mathrm{C}$ for $2 \mathrm{~h}$, and the conductivity value was measured using a conductivity meter (Fisher Scientific) and signed as EL1. Then the sample was boiled at $95{ }^{\circ} \mathrm{C}-100{ }^{\circ} \mathrm{C}$ for 20 mins, and the conductivity value (EL2) was measured after the sample reached room temperature.

\section{Statistical analysis}

Student's t-test was used to determine the statistically significant difference between the means from different data groups. $P<0.05$ was statistically significant and marked as *. $P<0.01$ was statistically highly significant and marked as $*$.

\section{Additional files}

Additional file 1: Table S1. GhLRR-RLKs gene list. Table S2. Gene Localization and duplication Data. Table S3. InterProScan analysis. Table S4. Motif composition for each subclade. Table S5. List of SRA datasets downloaded for expression analysis. Table S6. Expression analysis. (XLSX $857 \mathrm{~kb}$ )

Additional file 2: Data S1. Protein alignment of AtSIFs and GhLRR-RLKs. (PDF $2711 \mathrm{~kb}$ )

Additional file 3: Figure S1. GhLRR-RLKs Chromosomal distribution. Figure S2. Exon-intron analysis of GhLRR-RLKs. Figure S3. Protein size distribution analysis of GhLRR-RLK. Figure S4. InterProScan domains distribution of GhLRR-RLKs. Figure S5. Protein structure and domain composition of GhLRR-RLKs. Figure S6. Extracellular motif composition. Figure S7. Blas $2 \mathrm{GO}$ annotation statistics. Figure S8. Cellular component analysis of GhLRR-RLKs. Figure S9. Biological processes analysis of GhLRR$R L K s$. Figure S10. Molecular function analysis of GhLRR-RLKs. Figure S11. Expression analysis of GhLRR-RLKs. (PPTX $7480 \mathrm{~kb}$ )

\section{Abbreviations}

AA: Amino acid residues; BAK1: Brassinosteroid Insensitive 1-associated receptor kinase 1; BKK1: BAK1-Like 1; CDS: Coding Sequence; CR4-class: CRINKLY4 class RLK; DPA: Day post anthesis; FLS2: Flagellin Sensitive 2; KD: Protein kinase-like domain; LRR-RLKs: Leucine Rich Repeats-RLKs; MEGA: Molecular Evolutionary Genetics Analysis; NJ: Neighbor-Joining; pl: Isoelectric point; PR5-RLK: PR5-Like RLK; RLKs: Receptor-like protein kinases; SERKs: Somatic Embryogenesis Receptor Kinase; SIF: Stress Induced Factor; SRA: Short Read Archive; S-RLK: S-domain RLK; TDE: Tandem duplication event; TRV: Tobacco Rattle Virus; UTR: Untranslated Region; VIGS: Virus-induced gene silencing; WAK: Wall Associated Kinase

\section{Acknowledgements}

We would like to thank the department of Plant and Sciences for partially supporting the study.

\section{Funding}

This research described in this manuscript was partially supported by Cotton Incorporated Core Project No. 18-092. The funding agency had no role in study design, data collection and analysis, or preparation of the manuscript.

\section{Availability of data and materials}

The datasets used and/or analyzed during the current study are available from the corresponding author on reasonable request.

\section{Authors' contributions}

VM conceived the study and designed the experiments. NY, KMR and VM performed the bioinformatic analysis and NY performed the gene functional characterization. NY, KMR, SKU, HL, VB and VM wrote the manuscript. All authors read and approved the final manuscript.

Ethics approval and consent to participate

Not applicable.

\section{Consent for publication}

Not applicable.

\section{Competing interests}

The authors declare that they have no competing interests.

\section{Publisher's Note}

Springer Nature remains neutral with regard to jurisdictional claims in published maps and institutional affiliations.

\section{Author details}

${ }^{1}$ Fiber and Biopolymer Research Institute (FBRI), Department of Plant and Soil Science, Texas Tech University, Lubbock, TX 79409, USA. ${ }^{2}$ Department of Botany, Panjab University, Chandigarh 160014, India. ${ }^{3}$ Department of Genetics and Biochemistry, Clemson University, Clemson, SC 29634, USA. 
Received: 2 May 2018 Accepted: 27 August 2018 Published online: 06 September 2018

\section{References}

1. Medzhitov R. Toll-like receptors and innate immunity. Nat Rev Immunol. 2001;1(2):135-45.

2. Torii KU. Leucine-rich repeat receptor kinases in plants: structure, function, and signal transduction pathways. Int Rev Cytol. 2004;234:1-46.

3. Becraft PW, Stinard PS, McCarty DR. CRINKLY4: a TNFR-like receptor kinase involved in maize epidermal differentiation. Science. 1996;273(5280):1406-9.

4. Pastuglia M, RuffioChable V, Delorme V, Gaude T, Dumas C, Cock JM. A functional $\mathrm{S}$ locus anther gene is not required for the self-incompatibility response in Brassica oleracea. Plant Cell. 1997;9(11):2065-76.

5. McCarty DR, Chory J. Conservation and innovation in plant signaling pathways. Cell. 2000;103(2):201-9.

6. He Z, Fujiki M, Kohorn B. A cell wall-associated, receptor-like protein kinase. J Biol Chem. 1996:271(33):19789-93.

7. Miller D, Hable W, Gottwald J, Ellard-Ivey M, Demura T, Lomax T, Carpita N. Connections: the hard wiring of the plant cell for perception, signaling, and response. Plant Cell. 1997;9(12):2105-17.

8. Wang $X$, Zafian $P$, Choudhary M, Lawton M. The PR5K receptor protein kinase from Arabidopsis thaliana is structurally related to a family of plant defense proteins. P Natl Acad Sci USA. 1996;93(6):2598-602.

9. Jones DA, Jones JDG. The role of leucine-rich repeat proteins in plant defences. Adv Bot Res. 1997;24:89-167.

10. Gish LA, Clark SE. The RLK/Pelle family of kinases. Plant J. 2011;66(1):117-27.

11. Wu Y, Xun Q, Guo Y, Zhang J, Cheng K, Shi T, He K, Hou S, Gou X, Li J. Genome-wide expression pattern analyses of the Arabidopsis leucine-rich repeat receptor-like kinases. Mol Plant. 2016;9(2):289-300.

12. Gou X, He K, Yang H, Yuan T, Lin H, Clouse SD, Li J. Genome-wide cloning and sequence analysis of leucine-rich repeat receptor-like protein kinase genes in Arabidopsis thaliana. BMC Genomics. 2010;11:19.

13. Sun Y, Li L, Macho AP, Han Z, Hu Z, Zipfel C, Zhou JM, Chai J. Structural basis for flg22-induced activation of the Arabidopsis FLS2-BAK1 immune complex. Science. 2013;342(6158):624-8.

14. Nam KH, Li J. BRI1/BAK1, a receptor kinase pair mediating brassinosteroid signaling. Cell. 2002;110(2):203-12.

15. Deeken R, Kaldenhoff R. Light-repressible receptor protein kinase: a novel photo-regulated gene from Arabidopsis thaliana. Planta. 1997;202(4):479-86.

16. Li J, Chory J. A putative leucine-rich repeat receptor kinase involved in brassinosteroid signal transduction. Cell. 1997;90(5):929-38.

17. Fletcher JC, Brand U, Running MP, Simon R, Meyerowitz EM. Signaling of cell fate decisions by CLAVATA3 in Arabidopsis shoot meristems. Science. 1999;283(5409):1911-4

18. de Lorenzo L, Merchan F, Laporte P, Thompson R, Clarke J, Sousa C, Crespi M. A novel plant leucine-rich repeat receptor kinase regulates the response of Medicago truncatula roots to salt stress. Plant Cell. 2009;21(2):668-80.

19. Xiang $Y$, Cao $Y, X u$ C, Li X, Wang S. Xa3, conferring resistance for rice bacterial blight and encoding a receptor kinase-like protein, is the same as Xa26. Theor Appl Genet. 2006;113(7):1347-55.

20. Albrecht C, Russinova E, Kemmerling B, Kwaaitaal M, de Vries SC. Arabidopsis SOMATIC EMBRYOGENESIS RECEPTOR KINASE proteins serve brassinosteroiddependent and -independent signaling pathways. Plant Physiol. 2008;148(1): 611-9.

21. Gou X, Yin H, He K, Du J, Yi J, Xu S, Lin H, Clouse SD, Li J. Genetic evidence for an indispensable role of somatic embryogenesis receptor kinases in brassinosteroid signaling. PLoS Genet. 2012;8(1):e1002452.

22. Roux M, Schwessinger B, Albrecht C, Chinchilla D, Jones A, Holton N, Malinovsky FG, Tor M, de Vries S, Zipfel C. The Arabidopsis leucine-rich repeat receptor-like kinases BAK1/SERK3 and BKK1/SERK4 are required for innate immunity to hemibiotrophic and biotrophic pathogens. Plant Cell. 2011:23(6):2440-55.

23. Wang GL, Ruan DL, Song WY, Sideris S, Chen L, Pi LY, Zhang S, Zhang Z, Fauquet C, Gaut BS, et al. Xa21D encodes a receptor-like molecule with a leucine-rich repeat domain that determines race-specific recognition and is subject to adaptive evolution. Plant Cell. 1998;10(5):765-79.

24. Zhou F, Guo Y, Qiu LJ. Genome-wide identification and evolutionary analysis of leucine-rich repeat receptor-like protein kinase genes in soybean. BMC Plant Biol. 2016;16:58.
25. Magalhaes DM, Scholte LL, Silva NV, Oliveira GC, Zipfel C, Takita MA, De Souza AA. LRR-RLK family from two Citrus species: genome-wide identification and evolutionary aspects. BMC Genomics. 2016;17(1):623.

26. Zhu H, Wang Y, Yin H, Gao M, Zhang Q, Chen Y. Genome-wide identification and characterization of the LRR-RLK gene family in two Vernicia species. Int J Genomics. 2015;2015:823427.

27. Song W, Wang B, Li X, Wei J, Chen L, Zhang D, Zhang W, Li R. Identification of immune related LRR-containing genes in maize (Zea mays L.) by genome-wide sequence analysis. Int J Genomics. 2015;2015:231358.

28. Sun X, Wang GL. Genome-wide identification, characterization and phylogenetic analysis of the rice LRR-kinases. PLoS One. 2011;6(3):e16079.

29. Petre B, Hacquard S, Duplessis S, Rouhier N. Genome analysis of poplar LRRRLP gene clusters reveals RISP, a defense-related gene coding a candidate endogenous peptide elicitor. Front Plant Sci. 2014;5:111.

30. Shumayla SS, Kumar R, Mendu V, Singh K, Upadhyay SK. Genomic dissection and expression profiling revealed functional divergence in Triticum aestivum Leucine Rich Repeat Receptor Like Kinases (TaLRRKs). Front Plant Sci. 2016;7:1374

31. Chen CW, Panzeri D, Yeh YH, Kadota Y, Huang PY, Tao CN, Roux M, Chien SC, Chin TC, Chu PW, et al. The Arabidopsis malectin-like leucine-rich repeat receptor-like kinase IOS1 associates with the pattern recognition receptors FLS2 and EFR and is critical for priming of pattern-triggered immunity. Plant Cell. 2014;26(7):3201-19.

32. Yuan N, Yuan S, Li Z, Zhou M, Wu P, Hu Q, Mendu V, Wang L, Luo H. STRESS INDUCED FACTOR 2, a leucine-rich repeat kinase regulates basal plant pathogen defense. Plant Physiol. 2018;176(4):3062-80.

33. Chen ZJ, Scheffler BE, Dennis E, Triplett BA, Zhang T, Guo W, Chen X, Stelly DM, Rabinowicz PD, Town CD, et al. Toward sequencing cotton (Gossypium) genomes. Plant Physiol. 2007;145(4):1303-10.

34. Li Y, Sun J, Xia G. Cloning and characterization of a gene for an LRR receptor-like protein kinase associated with cotton fiber development. Mol Gen Genomics. 2005;273(3):217-24.

35. Jun Z, Zhang Z, Gao Y, Zhou L, Fang L, Chen X, Ning Z, Chen T, Guo W, Zhang T. Overexpression of GbRLK, a putative receptor-like kinase gene, improved cotton tolerance to Verticillium wilt. Sci Rep. 2015;5:15048.

36. Sun Y, Fokar M, Asami T, Yoshida S, Allen RD. Characterization of the brassinosteroid insensitive 1 genes of cotton. Plant Mol Biol. 2004;54(2):221-32.

37. Xiao Y, Luo M, Hou L, Luo K, Luo X, Pei Y. Cloning and characterization of a LRR resistance like (GhLRR-RL) protein gene from cotton (Gossypium hirsutum L.). Acta Genet Sin. 2002;29(7):653-8.

38. Lehti-Shiu MD, Zou C, Hanada K, Shiu SH. Evolutionary history and stress regulation of plant receptor-like kinase/pelle genes. Plant Physiol. 2009;150(1):12-26.

39. Helft L, Reddy V, Chen X, Koller T, Federici L, Fernandez-Recio J, Gupta R, Bent A. LRR conservation mapping to predict functional sites within protein leucine-rich repeat domains. PLoS One. 2011;6(7):e21614.

40. Götz S, García-Gómez JM, Terol J, Williams TD, Nagaraj SH, Nueda MJ, Robles M, Talón M, Dopazo J, Conesa A. High-throughput functional annotation and data mining with the Blast2GO suite. Nucleic Acids Res. 2008;36(10):3420-35.

41. Stein MA, Leung K, Zwick M, Portillo FG, Finlay BB. Identification of a Salmonella virulence gene required for formation of filamentous structures containing lysosomal membrane glycoproteins within epithelial cells. Mol Microbiol. 1996; 20(1):151-64

42. Hayward A, Padmanabhan M, Dinesh-Kumar SP. Virus-induced gene silencing in nicotiana benthamiana and other plant species. Methods Mol Biol. 2011;678:55-63.

43. Taibi K, Taibi F, Abderrahim LA, Ennajah A, Belkhodja M, Mulet JM. Effect of salt stress on growth, chlorophyll content, lipid peroxidation and antioxidant defence systems in Phaseolus vulgaris L. S Afr J Bot. 2016;105:306-12.

44. Demidchik V, Straltsova D, Medvedev SS, Pozhvanov GA, Sokolik A, Yurin V. Stress-induced electrolyte leakage: the role of $\mathrm{K}+-$ permeable channels and involvement in programmed cell death and metabolic adjustment. J Exp Bot. 2014;65(5):1259-70.

45. Li FG, Fan GY, Lu CR, Xiao GH, Zou CS, Kohel RJ, Ma ZY, Shang HH, Ma XF, Wu JY, et al. Genome sequence of cultivated upland cotton (Gossypium hirsutum TM-1) provides insights into genome evolution. Nat Biotechnol. 2015;33(5):524-U242

46. Zhang TZ, Hu Y, Jiang WK, Fang L, Guan XY, Chen JD, Zhang JB, Saski CA Scheffler BE, Stelly DM, et al. Sequencing of allotetraploid cotton (Gossypium hirsutum L. acc. TM-1) provides a resource for fiber improvement. Nat Biotechnol. 2015;33(5):531-U252. 
47. Schallus T, Jaeckh C, Feher K, Palma AS, Liu Y, Simpson JC, Mackeen M, Stier G, Gibson TJ, Feizi T, et al. Malectin: a novel carbohydrate-binding protein of the endoplasmic reticulum and a candidate player in the early steps of protein N-glycosylation. Mol Biol Cell. 2008;19(8):3404-14.

48. Yeh Y, Panzeri D, Kadota Y, Huang Y, Huang P, Tao CN, Roux M, Chien H, Chin T, Chu P. The Arabidopsis malectin-like/LRR-RLK IOS1 is critical for BAK1-dependent and BAK1-independent pattern-triggered immunity. Plant Cell. 2016;28(7):1701-21.

49. Hok S, Danchin EG, Allasia V, Panabieres F, Attard A, Keller H. An Arabidopsis (malectin-like) leucine-rich repeat receptor-like kinase contributes to downy mildew disease. Plant Cell Environ. 2011;34(11):1944-57.

50. Schwessinger B, Roux M, Kadota Y, Ntoukakis V, Sklenar J, Jones A, Zipfel C. Phosphorylation-dependent differential regulation of plant growth, cell death, and innate immunity by the regulatory receptor-like kinase BAK1. PLoS Genet. 2011:7(4):e1002046.

51. Yu J, Jung S, Cheng CH, Ficklin SP, Lee T, Zheng P, Jones D, Percy RG, Main D. CottonGen: a genomics, genetics and breeding database for cotton research. Nucleic Acids Res. 2014;42(Database issue):D1229-36.

52. Lamesch P, Berardini TZ, Li D, Swarbreck D, Wilks C, Sasidharan R, Muller R, Dreher K, Alexander DL, Garcia-Hernandez M, et al. The Arabidopsis Information Resource (TAIR): improved gene annotation and new tools. Nucleic Acids Res. 2012;40(Database issue):D1202-10.

53. Finn RD, Clements J, Eddy SR. HMMER web server: interactive sequence similarity searching. Nucleic Acids Res. 2011;39(suppl_2):W29-37.

54. Marchler-Bauer A, Derbyshire MK, Gonzales NR, Lu S, Chitsaz F, Geer LY, Geer RC, He J, Gwadz M, Hurwitz DI, et al. CDD: NCBI's conserved domain database. Nucleic Acids Res. 2015;43(Database issue):D222-6.

55. Krogh A, Larsson B, von Heijne G, Sonnhammer EL. Predicting transmembrane protein topology with a hidden Markov model: application to complete genomes. J Mol Biol. 2001;305(3):567-80.

56. Tamura K, Stecher G, Peterson D, Filipski A, Kumar S. MEGA6: molecular evolutionary genetics analysis version 6.0. Mol Biol Evol. 2013;30(12): 2725-9.

57. Dereeper A, Audic S, Claverie JM, Blanc G. BLAST-EXPLORER helps you building datasets for phylogenetic analysis. BMC Evol Biol. 2010;10:8.

58. Hu B, Jin J, Guo AY, Zhang H, Luo J, Gao G. GSDS 2.0: an upgraded gene feature visualization server. Bioinformatics. 2015;31(8):1296-7.

59. Bailey TL, Gribskov M. Combining evidence using $p$-values: application to sequence homology searches. Bioinformatics. 1998;14(1):48-54

60. Petersen TN, Brunak S, von Heijne G, Nielsen H. SignalP 4.0: discriminating signal peptides from transmembrane regions. Nat Methods. 2011;8(10):785-6.

61. Livak KJ, Schmittgen TD. Analysis of relative gene expression data using real-time quantitative PCR and the $2-\Delta \Delta C T$ method. Methods. 2001;25(4): 402-8.

62. Liu Y, Schiff M, Dinesh-Kumar S. Virus-induced gene silencing in tomato. Plant J. 2002;31(6):777-86.

63. Gao X, Shan L. Functional genomic analysis of cotton genes with agrobacterium-mediated virus-induced gene silencing. Methods Mol Biol. 2013;975:157-65.

\section{Ready to submit your research? Choose BMC and benefit from:}

- fast, convenient online submission

- thorough peer review by experienced researchers in your field

- rapid publication on acceptance

- support for research data, including large and complex data types

- gold Open Access which fosters wider collaboration and increased citations

- maximum visibility for your research: over $100 \mathrm{M}$ website views per year

At BMC, research is always in progress.

Learn more biomedcentral.com/submissions 